\title{
RSR13 e modificação alostérica da afinidade hemoglobina-oxigênio: abuso entre atletas
}

\author{
Antonio Claudio Lucas da Nóbrega \\ Departamento de Fisiologia e Farmacologia; Pós-Graduação de Cardiologia; \\ Disciplina de Medicina do Exercício e do Esporte - Universidade Federal Fluminense, Niterói, RJ
}

\section{RESUMO}

O ácido metilpropiônico (RSR13) é um modificador alostérico da hemoglobina, com a qual se liga de forma nãocovalente, diminuindo sua afinidade pelo oxigênio de modo dose-dependente e, conseqüentemente, aumentando a oxigenação periférica. O objetivo deste artigo é apresentar brevemente as evidências científicas acerca das características farmacológicas e funcionais, indicações médicas e efeitos adversos do uso do RSR13 por atletas, a mais recente alternativa de aumento artificial do desempenho. Estudos experimentais preliminares verificaram algum efeito positivo do RSR13 sobre a recuperação do miocárdio isquêmico e sobre a extensão da isquemia cerebral, mas as principais indicações estudadas atualmente são a cirurgia com hipotermia e cardioplegia durante circulação extracorpórea e o uso como agente coadjuvante potenciador da radioterapia para certos tumores sólidos. Somente um estudo em modelo canino mostrou aumento do consumo máximo de oxigênio em músculo isolado, não existindo evidências de que o RSR13 possa efetivamente melhorar o desempenho em humanos. Em realidade, já foram descritos efeitos adversos, como diminuição da perfusão sanguínea, elevação da pressão arterial e diminuição da função renal. Antecipando o potencial aumento da utilização do RSR13 por

Recebido em: 10/1/2002

Aceito em: 5/2/2002

Endereço para correspondência:

Prof. Dr. Antonio Claudio Lucas da Nóbrega

Editor-Chefe da RBME

Departamento de Fisiologia e Farmacologia

\& Pós-Graduação de Cardiologia

Universidade Federal Fluminense

Rua Professor Hernani Pires de Mello, 101

24210-130 - Niterói, RJ

E-mail: anobrega@urbi.com.br atletas, métodos já foram desenvolvidos para sua detecção em amostras de urina humana.

Palavras-chave: Atleta. Consumo de oxigênio. Contração muscular. Desempenho. Dopagem.

\section{ABSTRACT \\ RSR13 and allosteric change in the hemoglobin-oxygen afinity: abuse among athletes}

Methylpropionic acid (RSR13) is an allosteric hemoglobin modifier to which it binds in a non-covalent manner, reducing its affinity to oxygen in a dose-dependent fashion, and consequently, increasing peripheral oxygenation. The purpose of this article is to briefly present scientific evidence concerning pharmacological and functional characteristics, medical indications, and adverse effects of RSR 13 use by athletes, the most recent alternative to enhance performance artificially. Preliminary experimental studies have verified some positive effect of RSR13 on the recovery of ischemic myocardium and on the extension of cerebral ischemia. However, the main indications currently under study are surgery with hypothermia and cardioplegia during extra-corporeal circulation and the use as a radiotherapy enhancing agent for certain solid tumors. One single study with a canine model has shown increased maximal oxygen consumption in isolated muscles, there existing no evidence that RSR13 can effectively enhance performance in humans. Actually, adverse effects such as reduced blood perfusion, increased arterial blood pressure, and impaired renal function have been described. As an anticipation of the potential increased use of RSR13 by athletes, methods have been developed for its detection in human urine samples.

Key words: Athlete. Doping. Muscle contraction. Oxygen consumption. Performance. 


\section{INTRODUÇÃO}

Paralelamente aos avanços do conhecimento fisiológico e clínico do organismo do atleta e do desenvolvimento de tecnologias de equipamentos de sofisticação crescente, também prossegue a busca continuada por novas alternativas farmacológicas de melhoria do desempenho desportivo. O uso/abuso de drogas ergogênicas acompanha o ser humano desde os Jogos Olímpicos da Grécia Antiga e, desde o reinício das Olimpíadas Modernas, já registramos o uso de estimulantes, anabolizantes esteróides, analgésicos, narcóticos, diuréticos, betabloqueadores e diversos hormônios e agentes correlatos, como hormônio do crescimento (hGH), ACTH, insulina, IGF-1, eritropoetina (EPO), entre outros ${ }^{1}$. O interesse pelo uso de hormônios deve-se não só aos efeitos potencialmente ergogênicos, mas também ao fato de que a síntese destes compostos através da recombinação gênica dificulta muito a sua detecção em amostras de fluidos biológicos, uma vez que, desta forma, apresentam estrutura molecular idêntica àquela produzida endogenamente. Tem surpreendido a crescente disposição de atletas e pessoas associadas de arriscar cada vez mais a saúde em busca da vitória.

Enquanto liderava a Giro D'Italia 2001, o ciclista italiano Dario Frigo, vencedor da prova Paris-Nice e do Tour da Normandia, foi surpreendido por policiais com HemAssist ${ }^{2}$, uma substância substituta do sangue que aumenta a transferência de $\mathrm{O}_{2}$, mas que ainda não se encontra disponível comercialmente, nem mesmo clandestinamente pela internet. Somente os profissionais envolvidos nos ensaios clínicos em andamento têm acesso a esses medicamentos ainda experimentais. Esta questão atesta a agressividade com que se buscam drogas ergogênicas, indo além do estabelecido, experimentando substâncias ainda não conhecidas o suficiente do ponto de vista da relação risco/benefício.

As manobras para aumentar a transferência de $\mathrm{O}_{2}$ em esportes de longa duração iniciou-se com a autotransfusão sanguínea, quando um concentrado de hemácias do próprio atleta colhido três meses antes é reinfundido imediatamente antes da competição. Do ponto de vista farmacológico, observamos após 1987 o crescente uso de EPO, cuja função é aumentar a eritropoese, provocando aumento da massa de hemácias na circulação. A apreensão de drogas como o HemAssist na Europa exemplifica o conceito atual de manipulação da capacidade de transferências de $\mathrm{O}_{2}$ através do uso de substitutos sanguíneos, os quais podem ser de dois tipos: 1) carreadores de $\mathrm{O}_{2}$ baseados na hemoglobina $(\mathrm{Hb})$ recombinante, bovina ou humana, ou 2) perfluorocarbonos. Estes últimos são compostos químicos naturais, cujos átomos de hidrogênio foram substituídos por fluoreto, que é muito solúvel ao $\mathrm{O}_{2}$. Dentre os carreadores de $\mathrm{O}_{2}$ originados da $\mathrm{Hb}$, o RSR 13 encontra-se em fase avançada de estudos clínicos.

O objetivo deste artigo é apresentar brevemente as evidências científicas acerca das características farmacológicas e funcionais, indicações médicas e efeitos adversos do uso do RSR13 por atletas, a mais recente alternativa de aumento artificial do desempenho. A meta mais ampla é enfatizar o conceito de que o uso de tais substâncias visando desempenho ofende não somente a ética do esporte, mas também contraria os princípios éticos e legais do profissional médico envolvido no processo.

\section{ESTRUTURA QUÍMICA E CARACTERÍSTICAS BIOFÍSICAS}

O ácido (2-[4-[[(3,5-dimetilanilino)carbonil]metil]fenoxi]-2-metilpropiônico, cujo nome genérico é efaproxiral ou simplesmente RSR13, é um composto sintético (Allos Therapeutics, Denver, CO, EUA), análogo da droga clofibrato e modificador alostético da $\mathrm{Hb}$, com a qual se liga de forma não-covalente através de pontes de hidrogênio e interações hidrofóbicas com três das quatro subunidades, diminuindo sua afinidade pelo $\mathrm{O}_{2}^{3-5}$. Assim sendo, o RSR13 não altera a massa de hemácias, mas interfere na função da $\mathrm{Hb}$, desviando para a direita a curva de dissociação da oxi-hemoglobina $\left(\mathrm{HbO}_{2}\right)$ de forma dose-dependente ${ }^{6,7}$. Este efeito corresponde, até certo grau, ao aumento da temperatura, diminuição do $\mathrm{pH}$ e elevação da $\mathrm{pCO}_{2}$. Muito embora as características da cinética da $\mathrm{Hb}$ em relação à $\mathrm{pO}_{2}$ não permitam grande redução da oxigenação do sangue ao passar pelos alvéolos pulmonares na vigência de menor afinidade da $\mathrm{Hb}$ pelo $\mathrm{O}_{2}$, pode ocorrer diminuição da saturação da $\mathrm{HbO}_{2}{ }^{7}$. Por outro lado, o efeito da RSR13 aumenta a taxa de transferência capilar-tecido de $\mathrm{O}_{2}$ ao perfundir a periferia ${ }^{4,6}$.

\section{INDICAÇÕES MÉDICAS POTENCIAIS}

Considerando o efeito farmacológico do RSR13 de aumento da oxigenação tissular, a princípio tais medicamentos poderiam ser benéficos nas condições em que houvesse hipóxia, independente do mecanismo fisiopatogênico. Dessa forma, estudos preliminares utilizando modelos animais verificaram algum efeito positivo sobre a recuperação do miocárdio isquêmico ${ }^{8,9} \mathrm{e}$ sobre a extensão da isquemia cerebral em ratos ${ }^{10,11}$.

Entretanto, apenas duas linhas de investigação têm-se desenvolvido até a fase de experimentos com seres humanos. Uma focaliza indivíduos submetidos a cirurgia com hipotermia e cardioplegia durante circulação extracorpórea. A baixa temperatura desvia a curva da $\mathrm{HbO}_{2}$ para a esquerda, o que aumenta a afinidade da $\mathrm{Hb}$ pelo $\mathrm{O}_{2}$, difi- 
cultando a oxigenação tissular ${ }^{12,13}$. O objetivo do uso do RSR13 seria diminuir a necessidade de transfusões, em face da diminuição do estoque dos bancos de sangue e do aumento do conhecimento sobre os riscos da transfusão sanguínea ${ }^{6,14,15}$.

A outra linha de pesquisa verifica os efeitos do RSR13 como agente coadjuvante potenciador da radioterapia para certos tumores sólidos, como o glioblastoma multiforme ${ }^{16}$ e tumores pulmonares inoperáveis, excluindo os de pequena célula ${ }^{17}$. O RSR13 aumenta a oxigenação do tecido tumoral, facilitando a ação da radioterapia ${ }^{18}$. Os ensaios clínicos estão em andamento e objetivam responder se a radioterapia suplementada pelo RSR13 após indução quimioterápica pode retardar a progressão da doença e aumentar a sobrevida.

\section{EFEITOS ADVERSOS}

Os efeitos colaterais mais comumente descritos são relacionados a aspectos hemodinâmicos e à função renal. Do ponto de vista hemodinâmico observam-se os efeitos esperados auto-regulatórios do fluxo sanguíneo em vigência de aumento da pressão parcial de $\mathrm{O}_{2}$ tecidual, quais sejam, vasoconstrição arteriolar e consequiente redução do fluxo sanguíneo e aumento da resistência vascular periférica ${ }^{19}$. Caso esses efeitos fossem sustentados cronicamente, poderiam levar ao desenvolvimento de hipertensão arterial sistêmica e pulmonar ${ }^{20}$.

Em relação à função renal, observou-se aumento da creatinina sérica tanto em modelos experimentais ${ }^{21}$, quanto em ensaios clínicos ${ }^{6}$. O mecanismo responsável pela depressão da função renal envolve, provavelmente, hipóxia da medular do órgão reflexa à hiperóxia cortical induzida pelo RSR13, conforme demonstrado em modelo de insuficiência renal aguda em ratos $^{21}$.

É importante salientar que tais efeitos são aqueles efetivamente atribuídos ao RSR13 ao longo dos poucos anos de seu uso experimental e recentes testes em humanos, sendo que a transição entre a bancada do laboratório e o uso à beira do leito deve ser prolongada ${ }^{15}$. Dessa forma, é possível que sejam descritos outros efeitos adversos, inclusive letais, à medida que mais observações controladas sejam publicadas $^{14,20}$.

\section{RECURSO ERGOGÊNICO?}

Acontece historicamente com drogas utilizadas como doping. Os usuários baseiam-se na ação farmacológica por acreditar que determinada droga possui efeito ergogênico. Entretanto, em muitos casos, a droga exerce ação benéfica somente em situações de déficit funcional. Além disso, inexistem evidências científicas comprovando a ação ergogê- nica da droga, mesmo porque não se justifica, do ponto de vista ético, a realização de estudos experimentais com drogas em humanos sem um objetivo médico estabelecido, como, por exemplo, melhoria da qualidade de vida e da longevidade.

Apenas um estudo experimental desenvolvido pelo grupo do Dr. Peter Wagner, na Universidade da Califórnia em La Jolla, verificou aumento efetivo da oxigenação tecidual e do consumo máximo de $\mathrm{O}_{2}$ durante contrações musculares $^{22}$ com o uso de RSR13. Vale destacar que tal estudo utilizou uma preparação do músculo gastrocnêmio isolado de cães contraído através de eletroestimulação, ou seja, uma situação muito longe do exercício físico prolongado em humanos. Além disso, o mesmo laboratório verificou que o uso de RSR13 não acelera a cinética de consumo de $\mathrm{O}_{2}$ durante a transição repouso-contração (equivalente a 60$70 \%$ do consumo máximo de $\mathrm{O}_{2}$ ) na mesma preparação de músculo canino.

Independente da confirmação da existência de um efeito ergogênico do RSR13, atletas o estão utilizando. Portanto, como ocorre comumente, os laboratórios de análise química envolvidos com controle de doping já buscam sistemas de detecção do uso de tal medicamento. Breidbach e Catlin ${ }^{23}$ descreveram um método para detectar, na urina de humanos, íons do RSR13 com limite inferior de detecção de $2 \mathrm{ng} / \mathrm{mL}$. Esse método baseia-se na associação de cromatografia gasosa com espectrometria de massa por ionização de impacto de elétrons.

\section{CONCLUSÃO}

Embora teoricamente ergogênico para competições aeróbicas, não existem, até o momento, evidências de qualquer efeito facilitador da performance do RSR13 em humanos. Efeitos colaterais hemodinâmicos e renais foram descritos e já foi desenvolvido um método de detecção urinária de íons do RSR13. Profissionais do esporte, particularmente aqueles responsáveis pela saúde dos atletas, devem considerar o uso/abuso de tais substâncias como uma tendência atual em esportes de longa duração.

\section{REFERÊNCIAS}

1. De Rose EH, Nóbrega ACL. Doping na atividade desportiva. In: Lasmar NP, Camanho GL, Lasmar RCP, editores. Medicina do Esporte. Rio de Janeiro: Revinter, 2002:32-46.

2. Lindsey J. Cycling's doping time bomb. Bicycling: Daily: Racing News 2002 (www.bicycling.com).

3. Randad RS, Mahran MA, Mehanna AS, Abraham DJ. Allosteric modifiers of hemoglobin. 1. Design, synthesis, testing, and structure-allosteric activity relationship of novel hemoglobin oxygen affinity decreasing agents. J Med Chem 1991;34:752-7. 
4. Safo MK, Moure CM, Burnett JC, Joshi GS, Abraham DJ. High-resolution crystal structure of deoxy hemoglobin complexed with a potent allosteric effector. Protein Sci 2001;10:951-7.

5. Phelps Grella M, Danso-Danquah R, Safo MK, Joshi GS, Kister J, Marden M, Hofman SJ, Abraham DJ. Synthesis and structure-activity relationships of chiral allosteric modifiers of hemoglobin. J Med Chem 2000; 43:4726-37.

6. Wahr JA, Gerber M, Venitz J, Baliga N. Allosteric modification of oxygen delivery by hemoglobin. Anesth Analg 2001;92:615-20.

7. Eichelbronner O, Siellenkamper A, D'Almeida M, Ellis CG, Sibbald WJ, Chin-Yee IH. Effects of $\mathrm{FI}\left(\mathrm{O}_{2}\right)$ on hemodynamic responses and $\mathrm{O}_{2}$ transport during RSR13-induced reduction in $\mathrm{P}_{50}$. Am J Physiol 1999; 277(1 Pt 2):H290-8.

8. Woods JA, Storey CJ, Babcock EE, Malloy CR. Right-shifting the oxyhemoglobin dissociation curve with RSR13: effects on high-energy phosphates and myocardial recovery after low-flow ischemia. J Cardiovasc Pharmacol 1998;31:359-63.

9. Pagel PS, Hettrick DA, Montgomery MW, Kersten JR, Warltier DC. RSR13, a synthetic allosteric modifier of hemoglobin, enhances recovery of stunned myocardium in dogs. Adv Exp Med Biol 1998;454-52731.

10. Grocott HP, Bart RD, Sheng H, Muira Y, Steffen R, Pearlstein RD, Warner DS. Effects of a synthetic allosteric modifier of hemoglobin oxygen affinity on outcome from global cerebral ischemia in the rat. Stroke 1998; 29:1650-5.

11. Mackensen GB, Nellgard B, Sarraf-Yazdi S, Dexter F, Steffen RP, Grocott HP, Warner DS. Post-ischemic RSR13 amplifies the effect of dizocilpine on outcome from transient focal cerebral ischemia in the rat. Brain Res 2000; 853:15-21.

12. Steffen RP. Effect of RSR13 on temperature-dependent changes in hemoglobin oxygen affinity of human whole blood. Adv Exp Med Biol 1998;454:527-31.

13. Kilgore KS, Schwartz CF, Gallagher MA, Steffen RP, Mosca RS, Bolling SF. RSR13, a synthetic allosteric modifier of hemoglobin, improves myocardial recovery following hypothermic cardiopulmonary bypass. Circulation 1999;100(Suppl 19): II351-6.

14. Topfer LA, Hailey D. Oxygen carriers ("blood substitutes"). Issues Emerg Health Technol 2001;21:1-6.

15. Winslow RM. Blood substitutes. Curr Opin Hematol 2002;9:146-51.

16. Kleinberg L, Grossman AS, Piantadosi S, Pearlman J, Engelhard H, Lesser G, Ruffer J, Gerber M. Phase I trial to determin the safety, pharmacodynamics, and pharmacokinetics of RSR13, a novel radioenhancer, in newly diagnosed glioblastoma multiforme. J Clin Oncol 1999;17: 2593-603.

17. Kavanagh BD, Khandelwal SR, Schmidt-Ullrich RK, Roberts JD, Shaw EG, Pearlman AD, Venitz J, Dusenbery KE, Abraham DJ, Gerber MJ. A phase I study of RSR13, a radiation-enhancing hemoglobin modifier: tolerance of repeated intravenous doses and correlation of pharmacokinetics with pharmacodynamics. Int J Radiat Oncol Biol Phys 2001;49: 1133-9.

18. Amorino GP, Lee H, Holburn GE, Paschal CB, Hercules SK, Shyr Y, Steffen RP, Choy H. Enhancement of tumor oxygenation and radiation response by the allosteric effector. Radiat Res 2001;156:294-300.

19. Kunert MP, Liard JF, Abraham DJ. RSR-13, an allosteric effector of hemoglobin, increases systemic and iliac vascular resistance in rats. Am J Physiol 1996;271(2 Pt 2):H602-13.

20. Schummacker YO, Schmid A, Dinkelmann S, Berg A, Northoff H. Artificial oxygen carriers - The new doping threat in endurance sport? Int J Sports Med 2001;22:566-71

21. Burke TJ, Malhotra D, Sjapiro JI. Effects of enhanced oxygen release from hemoglobin by RSR13 in an acute renal failure model. Kidney Int 2001;60:1407-14.

22. Richardson RS, Tagore K, Haseler LJ, Jordan M, Wagner PD. Increased $\mathrm{VO}_{2}$ max with right-shifted $\mathrm{HB}-\mathrm{O}_{2}$ dissociation curve at a constant $\mathrm{O}_{2}$ delivery in dog muscle in situ. J Appl Physiol 1998;84:995-1002.

23. Breidbach A, Catlin DH. RSR13, a potential athletic performance enhancement agent: detection in urine by gas chromatography/mass spectrometry. Rapid Commun Mass Spectrom 2001;15:2379-82. 